\title{
Identifying patients' criteria for assessment of doctors on Polish physician rating websites
}

\author{
Radosław Tymiński, Michał Walczewski, Michał Wieczorek
}

Medical University of Warsaw

\begin{abstract}
Introduction. Increasingly popular physician ranking websites have lately become a significant factor in choosing a physician.

Aim. The aim of this study was to establish the criteria by which patients assessed doctors on PRWs and which of these criteria were the most crucial during the general assessment of the physicians.

Material and methods. Selected narrative comments from two Polish PRWs: znanylekarz.pl and rankinglekarzy. pl were analysed on the basis of the following criteria: kindness and propriety, punctuality, communication with patients, condition and equipment of a doctor's office, length of the appointment, cost of the medical advice.

Results. Out of 4375 eligible comments kindness and propriety was assessed most frequently (3012 comments, $68.85 \%$ ), next was communication, which was evaluated in 2343 comments (53.55\%). Amongst the 3012 comments with assessed kindness and propriety, $77.66 \%$ (2339 comments) were described positively. In the group of comments with positively evaluated kindness and propriety 2230 comments (95.34\%) were generally positive. Furthermore, communication with patient was assessed in 2343 comments and in 1827 cases (77.98\%) the assessment was positive. 1810 comments with positively evaluated communication were generally positive (99.07\%).

Conclusions. There is a connection between the patients' positive assessment and physician's kindness, personal culture and communication skills; if physicians focus on the aforementioned abilities, it might lead to better physician perception, higher effectiveness of treatment and the lower number of potential law suits.
\end{abstract}

Keywords: physician ranking sites, doctor assessment, patient satisfaction, soft skills.

\section{Introduction}

As of late, physician rating websites (PRWs) are becoming easily accessible and increasingly popular [1] as sources of information and comments on doctors and other health professionals [2, 3]. The number of assessed doctors is increasing [4], and in contrary to the anxiety expressed by the medical profession, the majority of comments is unambiguously positive [1, 5-7]. Moreover, various studies indicate that for many patients PRWs have become a significant factor in choosing a physician [8]. Likewise, similar changes can be noticed in Poland, where the Internet is starting to play an important part as a source of information on health, diseases, treatment, as well as the assistance in choosing a physician [9].

On the basis of the results of previous studies it can be assumed that the ability to establish a good relationship with a patient is significant [6]. In our opinion, although this thesis is true, it insufficiently describes dependencies between assessment of a doctor and his or her personality traits and communication skills. We state that both politeness and propriety as well as the skills in interpersonal communication are essential components of a good doctor-patient relationship. Proper relations between both sides have influence not only on patient's compliance [10] and treatment results, but also on how a physician is perceived. In other words, we assumed the hypothesis that general patients' 
assessment of doctors depends on the physicians' ability to build a relationship with patient.

The purpose of this study was to verify two important matters. The first aim of this study was to verify what exact features of a doctor or a medical service, apart from professional competence, determine if a patient's comment on physician is negative or positive in general.

Secondly, we wanted to establish whether the obligation imposed by the Polish legislator in Article 31 Paragraph 1 of the Medical Profession Act of 5 December 1996 to provide a patient with full medical information is justified by patients' actual expectations. Due to these regulations a doctor should inform a patient about: his or her state of health, diagnosis, proposed and available diagnostic and/or therapeutic methods, predictable results of application or abandonment of these methods, treatment results and prognosis.

In addition, our study should help identify areas where doctor-patient relationship difficulties are still persistent.

The accurate assessment of existing problems is going to be a first step in search of solutions, which may increase patient compliance, improve treatment results and consequently increase patients' subjective satisfaction.

\section{Material and methods}

Narrative comments from two Polish PRWs were selected: znanylekarz.pl and rankinglekarzy.pl.

The analysed comments consisted of:

- comments on internists and pediatricians retrieved from znanylekarz.pl;

- comments on internists and gynaecologists retrieved from rankinglekarzy.pl;

Two different PRWs and a number of physicians with different medical specialties (internal medicine, gynaecology, pediatrics) were chosen to verify whether the results for each PRW and each medical specialty were comparable. If the results could be confirmed, it would suggest they are not dependent on the medical specialty nor the PRW.

Because of the large number of comments on each group of medical specialists and our intention to analyse every comment in each group on both PRWs, the study was limited to the physicians (from public and private healthcare) who practice medicine in Warsaw and its neighborhoods.

The comments from doctor profiles were included based on the following conditions:
1. the doctor's profile appeared in PRW's search engine results after typing in (in Polish) phrases: "gynaecologist Warsaw", "Warsaw gynaecologist", "internist Warsaw", "Warsaw internist", "pediatrist Warsaw", "Warsaw pediatrist";

and

2. at least 10 comments were assigned to the doctor's profile by a website administrator.

Two of the authors independently analysed all the narrative comments from the doctor profiles which met the aforementioned requirements, provided the comment satisfied at least one criterion of comments analysis (CNCA, see "The criteria of narrative comments analysis").

Therefore the comments from the following doctor profiles were excluded from the study:

- the profile did not appear in search results after typing in the phrases mentioned above.

- the profile had less than 10 comments assigned. We resigned from the analysis of these comments in order to exclude random comments, those which seemed to be emotionally biased, those written by the physicians themselves, on their request or by their competitors.

After classification, the comments were reassessed and those not meeting the CNCA were rejected.

The excluded comments can be divided into three main groups:

1. comments containing technical data - these comments did not contain any assessment of a physician; they referred mainly to the issues like physician work hours, his or her current workplace or a phone number.

2. comments referring only to professional competence of the physician - these comments contained only the assessment of professional competence, including accuracy of diagnostic process and treatment. Comments made by patients assumed to have been unable to assess this sphere properly, due to the task being outside of their competence, were disqualified.

3. very brief comments - comments which did not contain any justification for included assessment.

The qualification process was presented on the Figure 1 .

All the comments qualified were analysed on the basis of the criteria of narrative comments analysis (CNCA), which had been developed after the initial analysis of 80 comments. We established the following criteria of comment analysis:

1. Kindness and propriety 


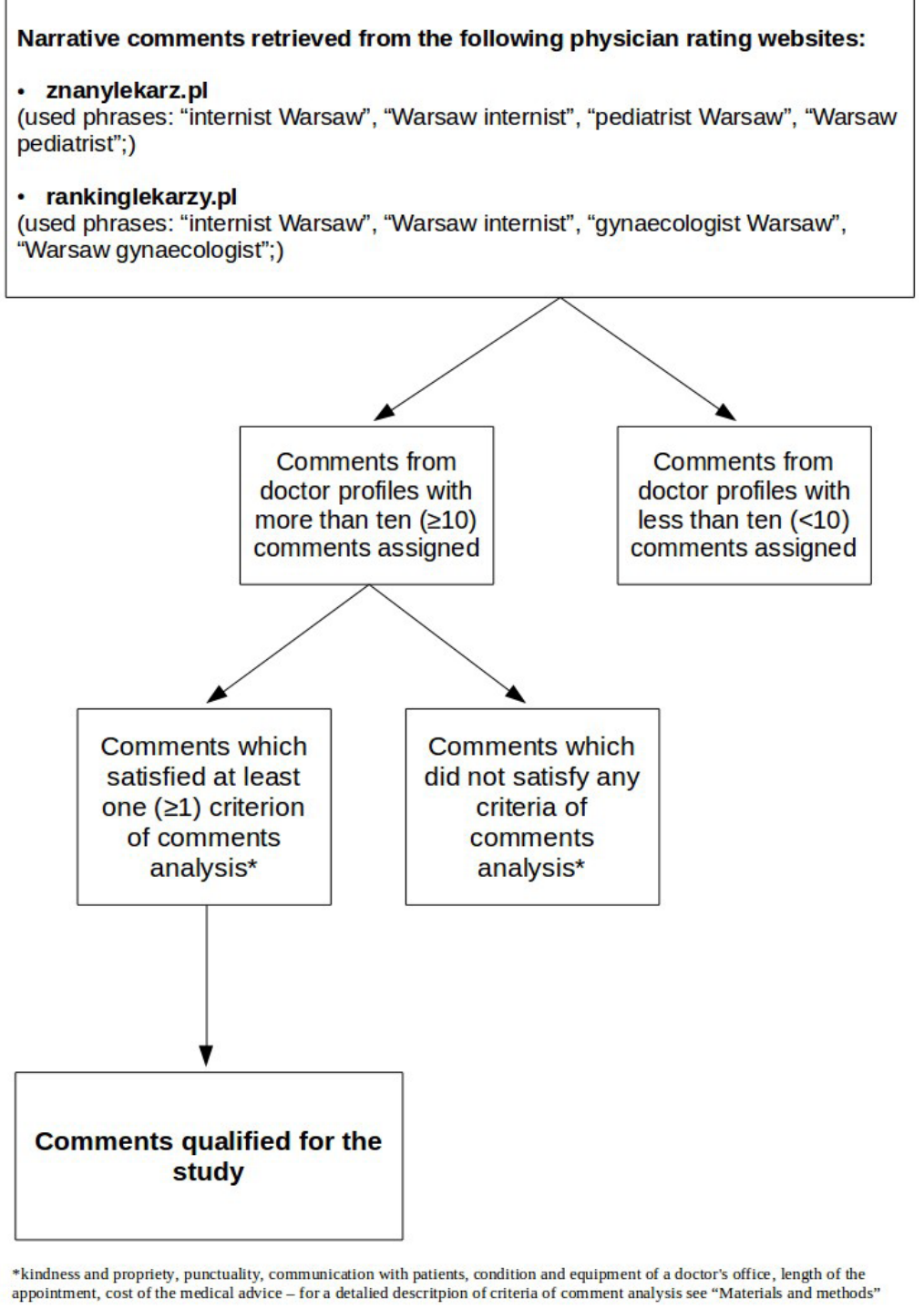

Figure 1. Qualification process

(Was the physician kind, polite, well-mannered, friendly, obliging, gentle, considerate?)

\section{Punctuality}

(Did the physician arrive at the consulting room on time?)

\section{Communication with patients}

(We assumed that communication was assessed in two situations: when the patient expressed his opinion on the general verbal and non-verbal communication skills of the physician [these were mostly the comments in which the patient stated whether physician talked with the patient, maintained eye contact] or when the patient described in depth doctor's communication skills, dividing them into one or more components [listening, explaining, asking questions]; in this case we assumed that the patient assessed general communication as well as in-depth communication).

\section{3a. Listening}

(Did the physician listen carefully to the patient?)

\section{3b. Explaining}

(Did the physician of their own will explain to the patient the pathophysiology of the disease? Did the doctor provide the patient with intelligible information about his or her state of health, diagnosis, proposed and available diagnostic and/or therapeutic methods, predictable results of application or abandonment of these methods, treatment results and prognosis.)

\section{3c. Asking questions}

(Did the patient have an opportunity to ask questions during the medical appointment? Did the physician answer those question?) 


\section{Condition and equipment of a doctor's office}

(Was the doctor's office clean? Was the equipment of good quality?)

5. Length of the appointment

(Was the patient satisfied with the length of the medical appointment?)

\section{Cost of the medical advice}

(Did the patient regard the cost of the medical advice as appropriate?)

Every qualified comment was analysed on the basis of the criteria mentioned above. If justification for the assessment which met the specific CNCA was found in a specific comment, then that assessment was qualified as positive or negative within a particular CNCA. It meant that the described by the specific CNCA aspect of medical practice was assessed respectively positively or negatively by the patient.

In our study we assumed that every comment could be qualified as:

- generally positive (a doctor who was assessed as very good or good on znanylekarz.pl or a doctor who received between $3,5 / 5$ and $5 / 5$ stars $(\leqslant 5$ and $>3,5$ stars) on rankinglekarzy.pl);

- generally negative (a doctor who was assessed as weak or very weak on znanylekarz.pl or a doctor who received between $1 / 5$ and $2,5 / 5$ stars $(\geqslant 1$ and $<2,5$ stars) on rankinglekarzy.pl);

- generally neutral (a doctor who was assessed as neutral on znanylekarz.pl or a doctor who received between $2,5 / 5$ and 3,5/5 stars ( $\leqslant 3,5$ and $\geqslant 2,5$ stars) on rankinglekarzy.pl).
If the comment was qualified to one of groups above, it meant that the general assessment of a physician in this comment was respectively positive, negative or neutral. As a result, the relation between the CNCA and a patient's general assessment of a doctor could be verified.

\section{Results}

From December 2014 to January 20154 groups of narrative comments were initially analysed: 2616 comments on 142 gynecologists and 560 comments on 36 internists from PRW rankinglekarzy.pl and also 2192 comments on 113 internists and 2321 comments on 121 pediatricians from PRW znanylekarz.pl . Altogether 4375 $(56,90 \%)$ comments met the criteria described in Methodology part and were included in the final analysis.

Out of 4375 eligible comments 3294 (75,39\%) were generally positive, 1002 (22,90\%) were negative and only 79 (1,81\%) were neutral.

Every narrative comment was analysed according to CNCA described in Methodology. Amongst all 4375 eligible comments most (3012 comments, 68,85\%) assessed kindness and propriety. Communication was evaluated in 2343 comments (53,55\%). Another most frequently assessed aspect of medical appointment was its length which was mentioned in $317 \mathrm{com}$ ments $(7,25 \%)$. It is necessary to emphasise that 759 $(41,93 \%)$ out of 1810 comments regarding communication in general were focused on a physician's ability to explain important matters concerning medical con-

Table 1. Results of particular categories divided into generally positive, negative and neutral comments

\begin{tabular}{|c|c|c|c|c|c|c|c|}
\hline \multirow{3}{*}{ Assessed element } & \multicolumn{7}{|c|}{ Number of concerns } \\
\hline & \multicolumn{2}{|c|}{$\begin{array}{l}\text { Generally positive comments } \\
\qquad(\mathrm{n}=3294)\end{array}$} & \multicolumn{2}{|c|}{$\begin{array}{l}\text { Generally negative comments } \\
\qquad(n=1002)\end{array}$} & \multicolumn{2}{|c|}{$\begin{array}{l}\text { Generally neutral comments } \\
\qquad(\mathrm{n}=79)\end{array}$} & \multirow{2}{*}{ Total } \\
\hline & $\begin{array}{l}\text { Assessed } \\
\text { positively }\end{array}$ & $\begin{array}{c}\text { Assessed } \\
\text { negatively }\end{array}$ & $\begin{array}{l}\text { Assessed } \\
\text { positively }\end{array}$ & $\begin{array}{l}\text { Assessed } \\
\text { negatively }\end{array}$ & $\begin{array}{l}\text { Assessed } \\
\text { positively }\end{array}$ & $\begin{array}{c}\text { Assessed } \\
\text { negatively }\end{array}$ & \\
\hline Kindness and propriety & 2230 & 29 & 75 & 630 & 34 & 14 & 3012 \\
\hline Punctuality & 12 & 7 & 0 & 30 & 0 & 2 & 51 \\
\hline $\begin{array}{l}\text { Communication with patient } \\
\text { (listening, explaining, asking } \\
\text { questions) }\end{array}$ & 1810 & 23 & 7 & 462 & 10 & 31 & 2343 \\
\hline Listening & 337 & 1 & 5 & 61 & 3 & 3 & 410 \\
\hline Explaining & 759 & 6 & 1 & 102 & 2 & 8 & 878 \\
\hline Asking questions & 491 & 2 & 2 & 124 & 2 & 3 & 624 \\
\hline $\begin{array}{l}\text { Condition and equipment } \\
\text { of the doctor's office }\end{array}$ & 18 & 1 & 1 & 6 & 1 & 0 & 27 \\
\hline Length of the appointment & 173 & 8 & 0 & 125 & 0 & 11 & 317 \\
\hline Cost of the medical advice & 21 & 14 & 0 & 32 & 0 & 6 & 73 \\
\hline Total & 5851 & 91 & 91 & 1572 & 52 & 78 & 7735 \\
\hline
\end{tabular}


ditions. They also mentioned the course of the appointment (Table 1).

In comments regarding internists and gynecologists, patients most often assessed kindness and propriety, then communication skills and then length of the appointment. By contrast, in comments concerning pediatricians communication with patient was the most frequently assessed aspect of the appointment, followed by kindness and propriety, and then length of the visit (Table 2).

Amongst the 3012 comments with assessed kindness and propriety, 77,66\% (2339 comments) were described positively. In the group of comments with positively evaluated kindness and propriety $2230 \mathrm{com}$ ments $(95,34 \%)$ were generally positive. Furthermore, communication with patient was assessed in 2343 comments and in 1827 cases $(77,98 \%)$ the assessment was positive. 1810 comments with positively evaluated communication were generally positive (99,07\%) (Figure 2).

\section{Discussion}

\section{Present literature}

Despite the growing popularity of PRWs amongst patients it appears that the researchers' interest in this subject is modest. On 15.10.2014 we searched the PubMed database using phrases "physician rating sites", "rating sites", "physician rating websites” and received 11, 20 and 23 search results respectively. In the Cochrane Library no results were found. Publications in the PubMed database focused e.g. on socio-

Table 2. Assessment of kindness and propriety and communication in different medical specialties. Generally neutral comments not included

\begin{tabular}{|c|c|c|c|c|c|}
\hline \multirow[b]{2}{*}{ Assessed element } & \multicolumn{2}{|c|}{ Generally positive comments } & \multicolumn{2}{|c|}{ Generally negative comments } & \multirow[b]{2}{*}{ Total } \\
\hline & $\begin{array}{l}\text { Assessed } \\
\text { positively }\end{array}$ & $\begin{array}{c}\text { Assessed } \\
\text { negatively }\end{array}$ & $\begin{array}{l}\text { Assessed } \\
\text { positively }\end{array}$ & $\begin{array}{c}\text { Assessed } \\
\text { negatively }\end{array}$ & \\
\hline \multicolumn{6}{|c|}{ Gynecologists, rankinglekarzy.pl } \\
\hline Kindness and propriety & 777 & 14 & 39 & 334 & 1164 \\
\hline Communication with patient & 579 & 8 & 4 & 205 & 796 \\
\hline \multicolumn{6}{|c|}{ Internists, rankinglekarzy.pl } \\
\hline Kindness and propriety & 158 & 0 & 1 & 74 & 233 \\
\hline Communication with patient & 103 & 0 & 0 & 47 & 150 \\
\hline \multicolumn{6}{|c|}{ Internists, znanylekarz.pl } \\
\hline Kindness and propriety & 722 & 6 & 17 & 85 & 830 \\
\hline Communication with patient & 507 & 9 & 1 & 80 & 597 \\
\hline \multicolumn{6}{|c|}{ Pediatricians, znanylekarz.pl } \\
\hline Kindness and propriety & 573 & 9 & 18 & 137 & 737 \\
\hline Communication with patient & 621 & 6 & 2 & 130 & 759 \\
\hline
\end{tabular}

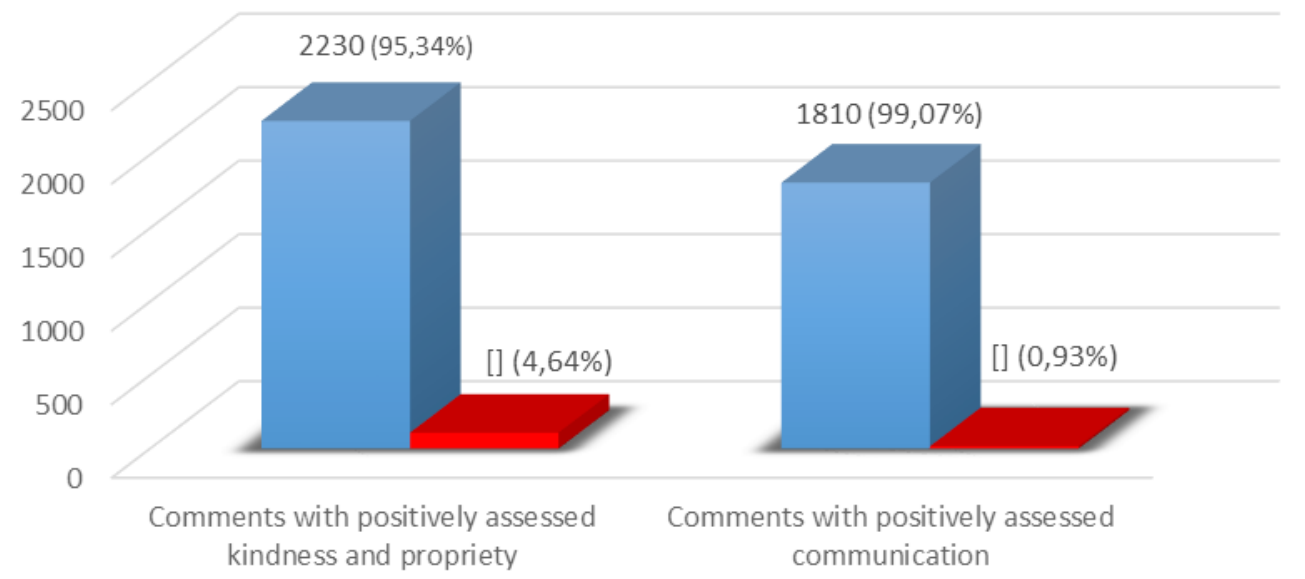

- Comments described as generally positive

Comments described as generally negative or neutral

Figure 2. Dependency between positive assessment of communication or kindness and the general evaluation of the doctor 
demographic analysis of PRW's users [24] or on the analysis of physicians' rating criteria on different websites [12]. Most of the studies we found originate from Germany, the United Kingdom and the United States of America. The majority of available literature was focused on scaled survey rating results [31, 32] and only few research papers concentrated on narrative comments analysis.

\section{Studies focused on narrative comments analysis}

During further online search, 5 studies concerning narrative comments analysis were retrieved. In the paper published in October 2014, Emmert et al. analysed 3000 narrative comments about German general practitioners and specialists [25]. Authors investigated 1500 randomly selected narrative comments on general practitioners and 1500 comments on specialists posted on the German PRS - jameda. Researchers evaluated patients' concerns mentioned in narrative comments and classified them into 50 different sub-categories from 3 main categories: physician, office staff, and practice. It is worth mentioning that the patient's perception of the physician's professional competence was included in the analysis.

The main goal of the American study conducted by Lopez et al. was to list all elements concerning medical appointment found during analysis of 712 randomly selected narrative comments posted on PRWs [26]. Greaves et al. and Alemi et al. evaluated American and British patients' online opinions using the artificial intelligence methods $[27,28]$. Lagu and colleagues analysed patients' text comments on hospitals posted in the first government-sponsored healthcare rating website - NHS Choices [29].

\section{Majority of comments are positive}

The results of our study are consistent with earlier research and confirms the previous conclusion that most comments published on PRWs are positive [1, $5-7,12,25]$. Our data presents that $77,39 \%$ from all eligible comments were positive. This particular observation is important in ongoing discussion with opponents of PRWs who claim, that physician rating websites are perfect platforms for defamation of doctors [12]. Interestingly enough, our results show that minority of comments are neutral $(1,81 \%)$ and these findings are consistent with the study of Emmert et al. [25]. That leads as to an important conclusion that physicians are assessed more frequently when patients are significantly satisfied, or on the contrary - significantly dissatisfied.

\section{Most frequently assessed aspects}

Outcomes of our study indicate, that regardless of doctor's specialization most of patients' concerns focus on: physician's kindness and propriety, communication with patient, length of the appointment. Those concerns occurred in $66,85 \%, 53,55 \%$ and $7,25 \%$ of all eligible comments respectively. According to Emmert et al. patients in PRWs most frequently describe physician's professional competence, then friendliness and caring attitude, and then time spent with the patient [25]. Information and communication are on $4^{\text {th }}$ and $7^{\text {th }}$ position in the list accordingly. As we can observe the main difference between our study and the German research lies in including patients' perception of physician's competence in the analysis. Presumably, other discrepancies in frequency of particular patient's concerns results from two issues. At first, it can be caused by differences between Polish and German patients. Secondly, our category "Communication with patient" is a combined point for plenty of aspects connected with communication (as described in "Methodology"). On the contrary, in the German study those aspects are separated in many different subcategories e.g. being taken seriously by a doctor or physician's child-friendliness.

Furthermore, analysis of differences in results between medical specialties demonstrates that pediatricians' communication with patients was evaluated more often than their kindness and propriety (Table 2). This discrepancy can be caused by the fact, that PRW users which comment on physicians from this exact specialty are mostly parents who pay heed to physician's communication with children and clear explanation of their child's medical condition.

\section{Key factor - communication}

As indicated before, patients in narrative comments posted in PRWs most frequently described kindness and propriety $(66,85 \%)$ which in $77,66 \%$ of cases was assessed positively. If kindness and propriety was assessed positively, 95,34\% of comments were generally positive (Figure 1). For communication this dependency was $99,07 \%$. In other words if physician's communication with a patient was assessed positively the predominant majority of comments were generally positive. These findings are substantial in the light of research signifying that proper doctor-patient communication results in improved patient's satisfaction from contact with a medical professional $[22,30]$. Fong $\mathrm{Ha}$ and Longnecker in their systematic review of literature pointed out studies which proved increased satisfac- 
tion from the medical appointment, abridged hospitalization, quicker convalescence, decreased pain sensation and better adherence to treatment in patients with good communication with a physician [30]. Bearing in mind this information, it is worth mentioning that public opinion poll carried out in 2014 indicates that only $54 \%$ of Polish people consider that patients in national healthcare system are treated with kindness [16].

\section{Significance of explanation}

Outcomes of our analysis demonstrate that amongst 1810 narrative comments concerning communication, most of the comments $(41,93 \%)$ were related to physician's willing explanation of diseases' patomechanisms and providing complete information about patient's medical condition, possible therapeutic options and their adverse effects (Table 1). Also in the German study cited above, information was the most frequently assessed subcategory related to communication [25]. Survey carried out amongst parents, whose children suffered from brachial plexus palsy during childbirth shows that poor communication and incomplete information correlated with more frequent malpractice litigations [17]. Moreover, previous research indicated that physicians who were sued more often, tended to get more complaints concerning their communication with patient $[18,19]$.

\section{Limitations of the study}

Major limitation of the study is that it concerns only online comments which are posted by a specific group of patients that uses PRWs and cannot perfectly represent the general population. Moreover we only addressed comments on 3 medical specialties and it is possible that there are significant differences in other specialties. Moreover we analysed comments from two major polish PRWs therefore there is a possibility that analysis of other websites can bring divergent results. Further research on this field are needed.

\section{Conclusion}

The conducted study reached designated goals. Although the study results cannot be completely extrapolated to the general patients' assessment of doctors, we claim that nevertheless the conclusions based on the studied PRWs are significant. Firstly it should be noticed that the majority of comments, which can be found on the studied PRWs, are positive and only few of them are neutral. Therefore PRWs can not be completely trusted as the source of information on a par- ticular physician, because the majority of patients, who have a broadly defined neutral opinion and are neither very satisfied nor disappointed with the doctor, probably do not use PRWs.

The most important achievement is that, in our opinion, we managed to prove a connection between the patients' positive assessment and physician's kindness, personal culture and communication skills. We believe that this conclusion is significant, because it clearly indicates that the positive assessment of a doctor depends on his or her relationship with a patient. Moreover it implies that physicians should attach more importance to socio-psychological aspects of their practice and therefore the development of soft skills should be a vital part of medical training. In addition, our study demonstrated that, in many comments, parents referred to the importance of a good relationship between a doctor and a child; this matter should be particularly important to pediatricians.

We proved that, for patients, the most important parts of communication with a doctor were detailed explanation and providing full medical information; it should be particularly noted that this conclusion is completely consistent with current legal situation, which requires doctors to provide a patient with full medical information.

Our findings allow us to present a hypothesis that there is a connection between the constantly increasing number of lawsuits against physicians based on the presumed or actual violation of patients' right to information, and the factual lack of full medical information in certain cases.

To sum up, kindness, politeness, and good communication with a patient might lead to better physician perception, higher effectiveness of treatment and the lower number of potential law suits. [17-19, 30].

\section{Acknowledgements}

The authors would like to thank Mr Jarosław Woś for language support.

\section{Conflict of interest statement}

The authors declare that there is no conflict of interest in the authorship or publication of contribution.

\section{Funding sources}

There are no sources of funding to declare.

\section{References}

1. Gao GG et al. A Changing Landscape of Physician Quality Reporting: Analysis of Patients' Online Ratings of Their Physicians Over a 5-Year Period. Journal of Medical Internet Research. 2012;14(1):e38. 
2. Verhoef LM et al. Social Media and Rating Sites as Tools to Understanding Quality of Care: A Scoping Review. Journal of Medical Internet Research. 2014;16(2):e56.

3. Mostaghimi A, Crotty BH, Landon BE. The Availability and Nature of Physician Information on the Internet. Journal of General Internal Medicine. 2010;25(11):11521156.

4. Hanauer DA et al. Public awareness, perception, and use of online physician rating sites. Jama. 2014;311(7): 734-5.

5. Emmert M, Meier F. An analysis of online evaluations on a physician rating website: evidence from a German public reporting instrument. J Med Internet Res. 2013;15(8): e157.

6. Lopez A et al. What patients say about their doctors online: a qualitative content analysis. J Gen Intern Med. 2012;27(6):685-92.

7. Kadry B et al. Analysis of 4999 online physician ratings indicates that most patients give physicians a favorable rating. J Med Internet Res. 2011;13(4):e95.

8. Emmert $\mathrm{M}$ et al. Physician choice making and characteristics associated with using physician-rating websites: cross-sectional study. J Med Internet Res. 2013;15(8): e187.

9. Polish Internet research. Online health websites. 2013.

10. Zolnierek KB, Dimatteo MR. Physician communication and patient adherence to treatment: a meta-analysis. Med Care. 2009;47(8):826-34.

11. Terlutter R, Bidmon S, Rottl J. Who uses physician-rating websites? Differences in sociodemographic variables, psychographic variables, and health status of users and nonusers of physician-rating websites. J Med Internet Res. 2014;16(3):e97.

12. Strech D, Reimann S. [German language physician rating sites]. Gesundheitswesen. 2012;74(8-9):e61-7.

13. Emmert M, Sander U, Pisch F. Eight questions about physician-rating websites: a systematic review. J Med Internet Res. 2013;15(2):e24.

14. Emmert $\mathrm{M}$ et al. What do patients say about their physicians? An analysis of 3000 narrative comments posted on a German physician rating website. Health Policy. 2014;118(1):66-73.

15. Greaves $\mathrm{F}$ et al. Use of sentiment analysis for capturing patient experience from free-text comments posted online. J Med Internet Res. 2013;15(11):e239.
16. Alemi $F$ et al. Feasibility of real-time satisfaction surveys through automated analysis of patients' unstructured comments and sentiments. Qual Manag Health Care. 2012;21(1):9-19.

17. Lagu $T$ et al. A mixed-methods analysis of patient reviews of hospital care in England: implications for public reporting of health care quality data in the United States. Jt Comm J Qual Patient Saf. 2013;39(1):7-15.

18. Levinson W, Hudak P, Tricco AC. A systematic review of surgeon-patient communication: strengths and opportunities for improvement. Patient Educ Couns. 2013;93(1): 3-17.

19. Ha JF, Longnecker N. Doctor-Patient Communication: A Review. The Ochsner Journal. 2010;10(1):38-43.

20. Public Opinion Research Center, Opinions About Healthcare System. 2014.

21. Domino J et al. Lack of physician-patient communication as a key factor associated with malpractice litigation in neonatal brachial plexus palsy. J Neurosurg Pediatr. 2014;13(2):238-42.

22. Levinson $W$ et al. Physician-patient communication. The relationship with malpractice claims among primary care physicians and surgeons. Jama. 1997;277(7):553-9.

23. Hickson GB et al. Obstetricians' prior malpractice experience and patients' satisfaction with care. Jama. 1994; 272(20):1583-7.

Acceptance for editing: 2015-02-10 Acceptance for publication: 2015-03-30

Correspondence address: Radosław Tymiński 9 tukowska Str, flat 126 04-133 Warsaw, Poland 